Indian Journal of Clinical Biochemistry, December 2007, Vol. 22 (Supl.)

\title{
LOCI ${ }^{\circledR}$ Technology: Making Light Work of Immunoassays
}

\author{
Martin A. Fuhrer
}

Director Global Marketing, Dade Behring Holding GmbH, Eschborn, Germany.

Key performance attributes for high sensitivity immunoassays are low background signal and broad dynamic measuring range. $\mathrm{LOCl}^{\circledR}$ technology provides these, as well as enabling small sample volumes, minimal instrument processing needs, and fast turnaround time. The reagent formulations are highly adaptable and may be configured in a variety of formats to meet the needs of a particular assay. This functionality makes $\mathrm{LOCl}^{\circledR}$ technology an excellent tool for the development of a broad range of homogeneous immunoassays. The technology uses two dye-containing synthetic bead reagents and a biotinylated analytespecific receptor. One bead reagent (sensibead) is coated with streptavidin and contains a photosensitive dye. The second bead reagent (chemibead) is coated with an assay binding partner and contains a chemiluminescent dye. During an assay, the three reactants combine with analyte to form a bead-aggregated immunocomplex. Illumination of the complex with light releases singlet oxygen from the sensibead, which diffuses into the chemibead and triggers chemiluminescence. $\mathrm{LOCl}^{\circledR}$ technology serves as the basis for a variety of sandwich and competitive format immunoassays on a new instrument system, the Dimension $V_{i s t a}{ }^{\circledR}$ system. The design and selected analytical performance data will be presented for various example methods to highlight the flexibility and capability of this technology. We conclude that use of $\mathrm{LOCl}^{\circledR}$ technology allows expedited development of immunoassays with a wide range of design needs. The technology is well suited to give high sensitivity detection, minimize interference from specimen components, and provide fast turnaround time in a variety of assay formats.

\section{Curriculum Vitae:}

Martin Fuhrer

After six years at the University Hospital of Tübingen, Germany as a Laboratory Supervisor, Martin joined DuPont in 1991 as Chemistry Applications and Sales Specialist with the Dimension and ACA product lines. Over the past 16 years he has covered various assignments including Training Specialist for Germany, Austria and Switzerland; Assistant Product Manager, Germany, Systems Specialist at the Dade Behring European Software Center, Senior Marketing Manager, International. In 2006 Martin returned from the USA where he was based for 3 years as Director of Global Marketing at the Dade Behring Global Chemistry Group headquarters in Glasgow, Delaware with main focus on Clinical Chemistry and Laboratory Automation. He is now Director Global Marketing Chemistry/Immunochemistry - European Branch based in Eschborn, Germany. 
Indian Journal of Clinical Biochemistry, December 2007, Vol. 22 (Supl.)

\section{Case-oriented overview and strategies for control of the Preanalytical Phase}

Sheshadri Narayanan

Weill Medical College Cornell University, New York, NY.

In an era where mechanization of laboratory automation has drastically reduced the errors due to the analytical phase of testing, errors due to the preanalytical phase are largely responsible for the decrease in quality of clinical laboratory results. Components of the preanalytical phase are so varied that at first sight the control of such multiple variables would appear to be daunting. Yet with increasing awareness of both physiological and controllable specimen collection, handling and processing errors and sample stability issues the contribution of preanalytical errors to the total quality of laboratory results can be significantly reduced. Rather than providing a didactic presentation I will instead approach the preanalytical phase in a case-oriented format and explore strategies for improving the quality of the preanalytical phase.

\section{References:}

1. Narayanan S. The Preanalytic Phase. An important component of laboratory medicine. Am J Clin Pathol 2000; 113:429-52

2. Guder WG, Narayanan S, Wisser H, Zawta B. Samples: From the patient to the laboratory: The impact of preanalytical variables on the quality of laboratory results. Wiley-VCH, Darmstadt, Germany, 2003, $3^{\text {rd }}$ edition

3. Narayanan S. Preanalytical issues in Hematology. J Lab Med 2003;27:243-8

4. Narayanan S, Young DS. Effect of Herbs and Natural Products on Clinical Laboratory Tests. AACC Press, Washington, DC, 2007

5. Young DS. Effect of Preanalytical variables on Clinical Laboratory Tests. AACC Press, Washington, DC, 2007, $3^{\text {rd }}$ edition

\section{Curriculum Vitae:}

\section{Sheshadri Narayanan}

She did her Ph.D. in Biochemistry from Graduate School of Basic Medical Sciences, New York in 1971. She is presently working as Adjunct Clinical Professor of Pathology and Laboratory Medicine, Weill Medical College of Cornell University. She is a member of many societies. She has got 3 books published along with more than 100 research papers in Indian and International journals. She has got one patent entitled "Blood Partitioning Composition" in 1991.

E-mail: Narayan_med_edu@msn.com

Phone: (212) 757 - 6748

Fax: (212) $414-7291$ 
Indian Journal of Clinical Biochemistry, December 2007, Vol. 22 (Supl.)

\section{Accreditation and Quality in Central Laboratory}

Anuradha Rajput

Director Central Laboratory Services - Clinigene Intl. Ltd. A Biocon Company

Meera Ghadge

Assistant Biochemist \& Deputy Quality Manager, Tata Memorial Hospital

The concept of centralized Laboratory:

Prior to 1986, laboratory testing for clinical trials was managed by clinical trial sponsors through the use of many different local or regional laboratories which increased the possibility for data errors, lengthened study timelines and increased study costs. Today, 100 of laboratory testing for clinical trials is outsourced. The analysis of clinical trials data often required months of work, with the data having to be "cleaned" first before it could be analyzed.

Burdened by the difficulties associated with gathering data from multiple local laboratories, which used different testing methodologies, reference ranges, and standard operation procedures. The idea for a central laboratory was conceived.

Today the concept of centralized laboratory services is well accepted

The industry has grown into a billion-dollar market that is now a critical component in more than 70 of all clinical trials worldwide

Quality in Clinical Laboratory:

Not many Big CROs/global pharmaceutical companies have set up their own facilities in India. They tend to use their facilities in Europe or the US for clinical trial purposes. Understandably, the investment in a centralized laboratory with good laboratory certification and national and international accreditation is huge and with the existing share of their business, probably the projected return on investment is not lucrative enough to invest in a dedicated central laboratory facility in India.

This has given rise to an opportunity for the local Clinical laboratories to scale up their facilities and investments to meet the global Quality standards

Accreditation of Clinical Laboratories:

Providing the best patient care requires laboratorians to strive for the highest performance. Excellence in our field isn't about the size of the laboratory or the title on the door. Knowledge and skill are important. Energy and persistence are important. So is Accreditation very Important. The accreditation programs examine all aspects of quality control and quality improvement in laboratory. including test methodologies, reagents, control media, equipment, specimen handling, procedure manuals, test reporting and internal and external proficiency testing and monitoring, as well as personnel, safety, and overall management practices that distinguish a quality laboratory.

Proficiency Testing: Laboratories should operate a quality assurance system which includes internal quality control procedures, participation in proficiency testing schemes, use of reference standards and certification/accreditation to a recognized standard such as ISO 15189/CAP. Proficiency testing schemes operate by providing participating laboratories with samples containing specified material but the actual quantity of the substance is known only to the organisers. The laboratory analyses the samples, preferably as part of their normal routine, and reports the results to the scheme organisers. The laboratory is then provided with a report showing how closely their results agree with the accepted value, and where necessary, can then take appropriate action to improve performance. Regular participation in a proficiency-testing 
Indian Journal of Clinical Biochemistry, December 2007, Vol. 22 (Supl.)

scheme provides independent verification of the analytical competence/Quality of a laboratory and shows a commitment to the maintenance and improvement of performance. It demonstrates to the public, customers, accreditation bodies, regulators, and management that analytical procedures are under control and gives analysts confidence that the service which they provide will withstand scrutiny.

- Case Study - Olympus AU 400.

\section{Curriculum Vitae:}

Anuradha Rajput

Director Central Laboratory Services -Clinigene Intl. Ltd. A Biocon Company

Dr Rajput has over 16 years of vast experience in Clinical Laboratory Management, both in hospital setting as well as in Clinical Research organization (CRO). She played a pivotal role in setting up the Central laboratory and the development of Quality systems at Clinigene. Dr. Rajput played a substantive role in getting CAP accreditation to Clinigene Central Laboratory, which happens to be the first laboratory in India with such accreditation. Prior to joining Clinigene Dr Rajput served as the head of Biochemistry Lab at Mallya Hospital and Manipal Hospital in Bangalore, India. Dr Rajput holds a PhD in Biochemistry from the University of Mysore. She is also a Qualified CAP Inspector. She has published her research work in peer- reviewed Scientific Journals \& is Board of Directors in one of the Diagnostic centers in Bangalore. She is also a member Board of studies in Bioscience at University of Mysore.

\section{Meera Ghadge}

Assistant Biochemist \& Deputy Quality Manager,Tata Memorial Hospital.

Dr. Meera Ghadge has a vast experience in Clinical Laboratory Management through her 24 years of association with Tata Memorial Hospital. She is instrumental in developing the Q.C systems in the department and ensuring NABL accreditation as per 15189 standards. Their Diagnostic Services Department holds the distinction of being the first among Public Organizations and the first among the departments in their hospital to be accredited for Quality Assurance. Dr. Ghadge holds a PhD in Biochemistry from the University of Mumbai .She had worked with M.D.Anderson Cancer Centre, Houston .She has a lot of publications to her credit and has been reviewing articles for IJCB. She holds the award from ACBI for the best paper published for the year 1996. 